\title{
Comparative Analysis of Pattern of Dermatological Disorders Treated During 2019 and 2020 at Benazir Bhutto Hospital Rawalpindi and Hospital Statistics: A Rapid Review of Effect of Covid-19
}

\author{
Rizwana Shahid ${ }^{1 *}$, Muhammad Umar ${ }^{2}$, Abdul Quddus Butt ${ }^{3}$ and Shawana Sharif ${ }^{4}$ \\ ${ }^{1}$ Assistant Professor Community Medicine, Rawalpindi Medical University, Pakistan \\ ${ }^{2}$ Vice Chancellor, Rawalpindi Medical University, Pakistan \\ ${ }^{3}$ Honorary Consultant Dermatologist, Benazir Bhutto Hospital, Rawalpindi, Pakistan \\ ${ }^{4}$ Assistant Professor Dermatology, Benazir Bhutto Hospital, Rawalpindi, Pakistan \\ *Corresponding author: Rizwana Shahid, Assistant Professor Community Medicine, Rawalpindi Medical University, Pakistan
}

\section{ARTICLE INFO}

Received: 幽 December 17, 2021

Published: 蔧 January 11, 2022

Citation: Rizwana Shahid, Muhammad Umar, Abdul Quddus Butt, Shawana Sharif. Comparative Analysis of Pattern of Dermatological Disorders Treated During 2019 and 2020 at Benazir Bhutto Hospital Rawalpindi and Hospital Statistics: A Rapid Review of Effect of Covid-19. Biomed J Sci \& Tech Res 41(1)-2022. BJSTR. MS.ID.006530.

Keywords: Dermatological Disorders; Erythroderma; Pemphigus Vulgaris; Eczema; Average Length Of Stay; Bed Occupancy Rate

Abbreviations: OPD: Outpatient Department; BOR: Bed Occupancy Rate; ALOS: Average Length of Stay; BBH: Benazir Bhutto Hospital; AQI: Air Quality Index; ALOS: Average Length of Stay
ABSTRACT

Objectives: To compare the pattern of dermatological ailments managed during 2019 and 2020 at Benazir Bhutto Hospital Rawalpindi and to assess hospital statistics.

Materials and Methods: A cross-sectional hospital record-based study was carried out by comparing the dermatological problems managed at BBH during 2019 and 2020. The data was collected regarding age, gender, number of patients visiting Out Patient Department (OPD), number of patients admitted and types of skin diseases among admitted. Data analysis was done by Microsoft Excel 2010. Bed Occupancy Rate (BOR) was computed. However, statistically significant gender wise differences in Average Length of Stay (ALOS) in hospital during 2019 were determined by Independent sample t-test. P-value $<0.05$ was considered significant. $95 \%$ CI was also calculated.

Results: About 63,649 and 75,280 patients with various skin diseases visited BBH during 2019 and 2020 respectively. Mean age of the patients was $39 \pm 7.2$ years. Females of 15-49 years age constituted the highest propensity of the sufferers. Approximately $65.8 \%$ and $76 \%$ females presented with skin problems at BBH during 2019 and 2020 respectively. About 185 dermatological patients got admitted at BBH during 2019 while 135 patients were admitted in 2020. Erythroderma was prevalent among $22.5 \%$ of the patients followed by pemphigus vulgaris (17.5\%) and eczema (15.6\%). Bed Occupancy Rate (BOR) was $49 \%$. Gender based differences in ALOS was statistically insignificant $(\mathrm{P}>0.60(-0.42-0.72))$.

Conclusion: Erythroderma was the commonest skin problem of our population. The drastic change was not reported in frequency of dermatological disorder amidst COVID pandemic. 


\section{Introduction}

About $1.9 \%$ billion of our global population is suffering from diverse skin problems [1]. Skin problems constitute the fourth commonest etiology of human ailments; about one third of global illness is attributed to dermatological diseases [2]. Apart from ethnicity and genetics, occurrence of skin disorders in any region are also attributed to dietary habits, hygienic practices, social class and climate [3]. COVID-19 has led to a very critical scenario across the globe due to confrontation with grave consequences. Apart from clinical symptoms suggestive of respiratory syndrome, SARS-CoV-2 also contributed to the development of depression and anxiety among our people [4]. Moreover, cutaneous lesions or changes have also determined now as one of the COVID related clinical presentations [5]. Pre-existing skin diseases like eczema and dermatitis among some patients got intensified due to COVID-19 [6]. A systematic review by Jamshidi et al revealed that presence of only dermatological lesions does not guarantee the severity of COVID-19 and patients with urticaria like manifestations had approximately $2.2 \%$ mortality rate [7].

The highest Disability Adjusted Life Years (DALY) rate for skin and subcutaneous infections was determined in New York; however, the difference in frequency of various dermatological problems between various American states was insignificant [8]. According to the results of screening program run among Germany inhabitants during 2019 , about $64.5 \%$ of the populates had skin disorders; the commonest among them was actinic keratosis (26.6\%) followed by rosacea (25.5\%) and eczema (11.7\%). Skin diseases were found to be more prevalent among elderly males [9]. The present study is intended to analyze the variations in frequency of skin problems by comparing the record of Dermatology department at BBH during 2019 and 2020. This will not only enable us to determine the differences in frequency of skin problems among our population during pre-COVID (2019) and COVID pandemic (2020); but will also facilitate us to comprehend the impact of COVID-19 on skin related ailments. This research will sufficiently guide our strategic planners towards taking necessary steps in order to combat COVID-19 associated skin disorders.

\section{Materials and Methods}

A cross-sectional hospital record-based study was done by comparing the dermatological problems managed at BBH during 2019 and 2020. The data was collected pertinent to age, gender, number of patients visiting OPD, number of patients admitted and type of skin disorders among admitted. Data was analyzed by Microsoft Excel 2010. Bed Occupancy Rate (BOR) was also computed. However, statistically significant gender-based variations in Average Length of Stay (ALOS) of the patients in hospital during 2019 and 2020 were determined by Independent sample t-test. P-value $<0.05$ was taken as significant. 95\% CI was also measured.

\section{Results}

A total of 63,649 and 75,280 patients with diverse dermatological problems visited Benazir Bhutto Hospital (BBH) Rawalpindi during 2019 and 2020 respectively. Mean age of the patients visiting the dermatology department both during 2019 \& 2020 was determined to be $39 \pm 7.2$ years. Gender-wise distribution of these patients is reflected below in Figure 1. Most of the patients presenting with skin diseases at BBH during 2019 and 2020 were 15-49 years old as illustrated below in Table 1. About 185 and 138 patients with dermatological problems were admitted in Dermatology ward of BBH through OPD and Accident \& Emergency department during 2019 and 2020 respectively. Gender based distribution of admitted patients is revealed below in Figure 2. Maximum number of patients 23 were admitted during July 2019 while least frequency of dermatological patients was reported in October 2019.

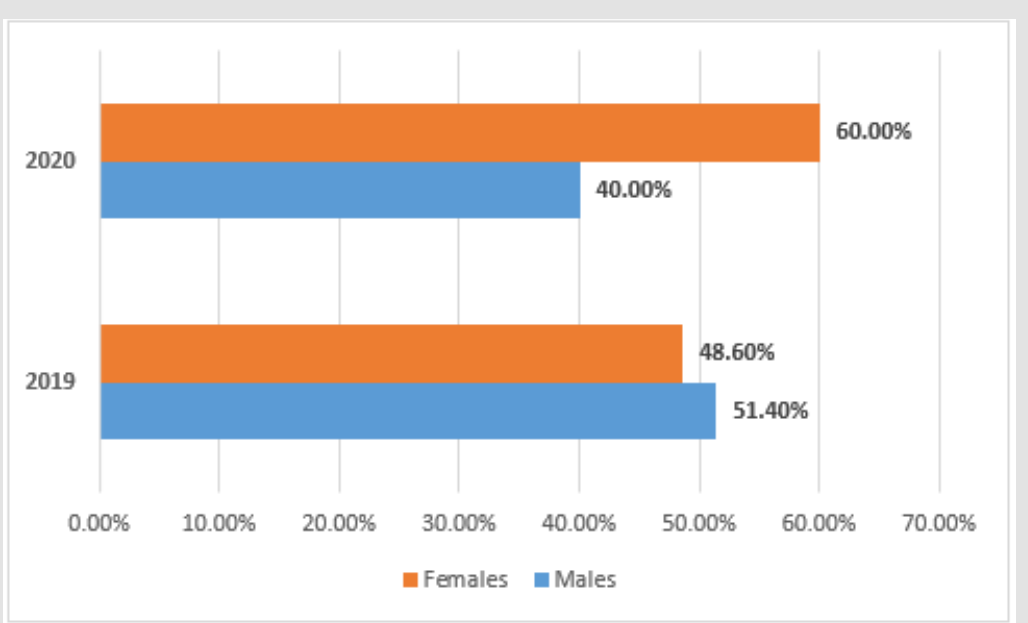

Figure 1: Gender based distribution of the patients with skin problems during 2019 and 2020. 


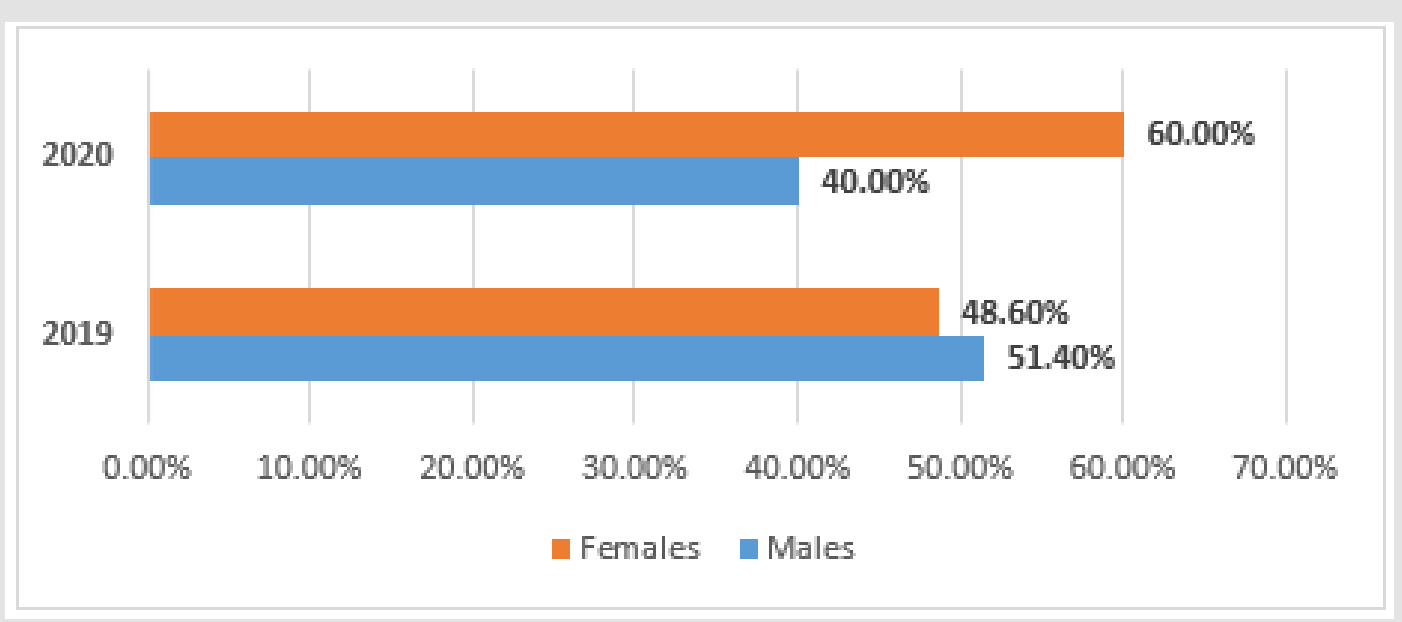

Figure 2: Gender wise differences in frequency of admissions during 2019 \& 2020.

Table 1: Age distribution of patients with skin problems.

\begin{tabular}{|c|c|c|c|c|}
\hline \multirow{2}{*}{ Age group } & \multicolumn{2}{|c|}{$\mathbf{2 0 1 9}$} & \multicolumn{2}{c|}{$\mathbf{2 0 2 0}$} \\
\cline { 2 - 5 } & Males & Females & Males & $1.20 \%$ \\
\hline$<1$ year & $2.10 \%$ & $1.80 \%$ & $1.83 \%$ & $4.60 \%$ \\
\hline $1-4$ years & $7.40 \%$ & $6.50 \%$ & $10.81 \%$ & $7.60 \%$ \\
\hline $5-14$ years & $17 \%$ & $15.30 \%$ & $19.90 \%$ & $76 \%$ \\
\hline $15-49$ years & $60 \%$ & $65.80 \%$ & $56.80 \%$ & $10.60 \%$ \\
\hline$\geq 50$ years & $13.50 \%$ & $10.60 \%$ & $10.66 \%$ & \\
\hline
\end{tabular}

On the other hand, highest number of admissions during 2020 was in the month of January and there was closure of Dermatology ward in April, May, June and July due to COVID pandemic as portrayed in Figure 3. The most commonly seen skin problem was erythroderma (22.5\%) among our patients followed by pemphigus vulgaris (17.5\%) and eczema (15.6\%). Top ten dermatological problems prevailing among our patients during 2019 and 2020 are depicted below in Figure 4. Bed Occupancy Rate was determined to be $49 \%$. Mean differences in ALOS of male and female dermatological patients was determined to be statistically insignificant as elucidated below in Table 2 .

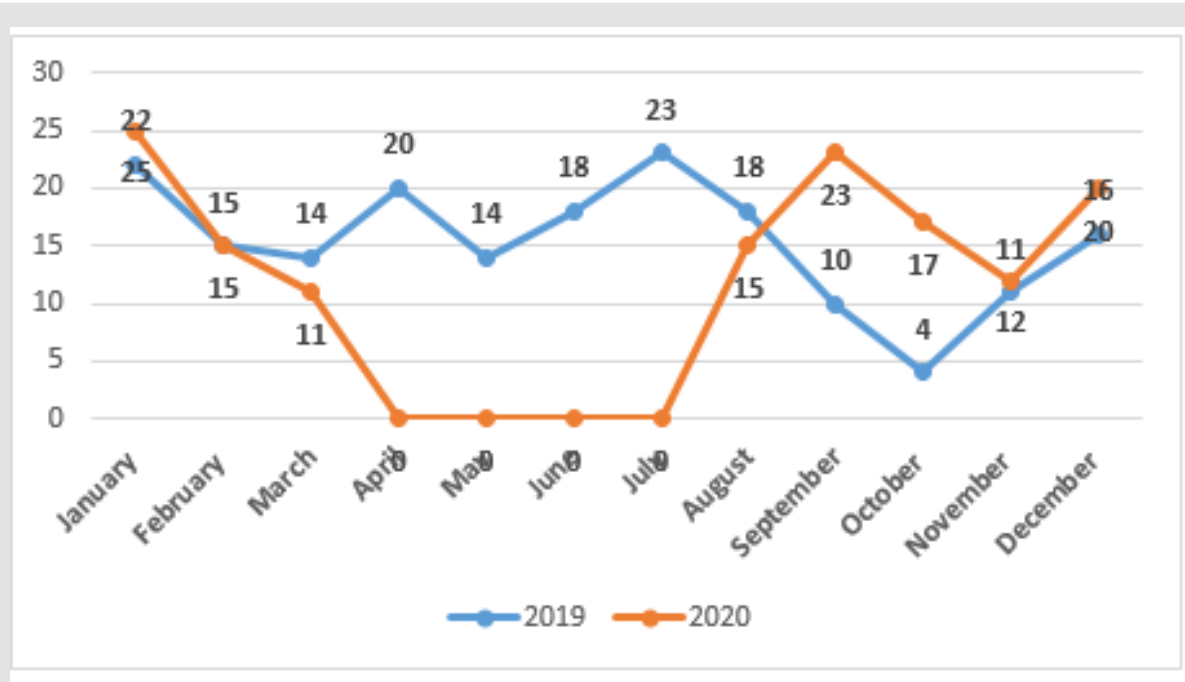

Figure 3: Trend of admissions in Dermatology ward during 2019 and 2020 at BBH. 


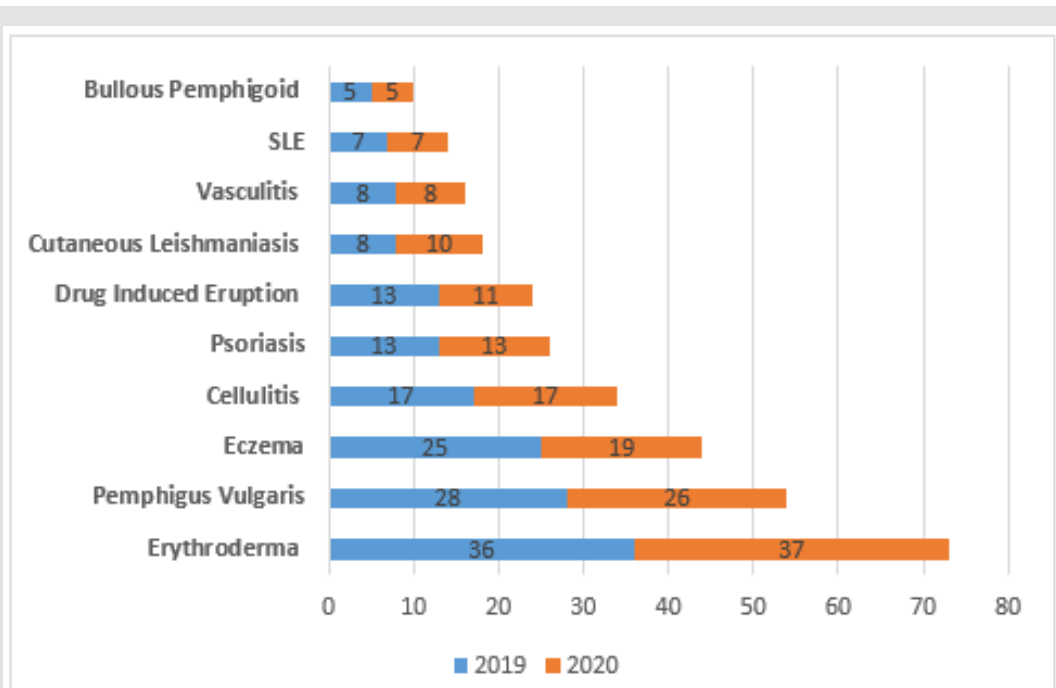

Figure 4: Top 10 dermatological problems reported at BBH during 2019 \& 2020.

Table 2: Statistical significance of gender-based variations in ALOS of admitted patients during $2019(\mathrm{n}=185)$.

\begin{tabular}{|c|c|c|c|}
\hline Attribute & Males (n= 95) & Females (n= 90) & P-value (95\% CI) \\
\hline ALOS & $4.25 \pm 2.14$ & $4.1 \pm 1.73$ & $0.60(-0.42-0.72)$ \\
\hline
\end{tabular}

\section{Discussion}

Skin diseases are frequently transmitted from infected individuals to the surrounding population. Comprehensive understanding of the global disease burden attributable to skin disorders is of paramount significance to mitigate cutaneous infections [10]. Skin problems can adequately be avoided by proper washing of hands, avoidance of contact with irritants and consultation with specialist in particular for chronic diseases [11].The present study revealed the maximum occurrence of skin problems among 15-49 years old females. The highest frequency (76\%) was among females who presented to Dermatology OPD of BBH during 2020 (Table 1). Although Dermatology department of Benazir Bhutto Hospital remained closed from April - July 2020, still patients visiting BBH were more $(75,280)$ than those $(63,649)$ who came to BBH with skin problems during 2019. This shows the confrontation of the population more with skin problems amidst COVID pandemic than during pre-pandemic era.

However, type of skin diseases remained same during both calendar years. A similar study carried out among dermatological patients attending primary healthcare facility of Karachi illustrated the mean age of the patients about 25 years; however, $87 \%$ of them were females. Approximately $43 \%$ had chronic skin ailments while $36.4 \%$ presented withinfectious disorders. Lifestylefactors thatwere significantly associated with onset of skin problems were sharing of fomites and contact with pet animals [12]. Role of lifestyle factors towards inception of skin problems should further be justified by doing systematic reviews. In ours study, erythroderma constituted the greatest magnitude of skin diseases both during 2019 and 2020 followed by pemphigus vulgaris, eczema and cellulitis. A similar cross-sectional study carried out in Tanzania revealed that eczema was the most frequent dermatological problem followed by papulosquamous disorders, fungal infections and tumors [13]. Likewise, a research among general public attending the Dermatology OPD of a tertiary healthcare facility of Lahore disclosed that most of the patients were 20-40 years old and eczema constituted the greatest magnitude $(31.07 \%)$ of dermatological illnesses. In addition, pigmented ailments were observed to be $3.82 \%$, psoriasis $3.80 \%$ and bullous disorders were $1.6 \%$ [14].

Skin problems among the residents of any geographical region vary from those residing in other territories; moreover, these differences also exist among people belonging to unlike age groups. In addition, the incidence of mortality from various skin disorders reflect much variation among developed and resource constrained countries [15]. A study by Huang $\mathrm{K}$ et al explored skin common diseases among Chinese inhabitants as both malignant and benign tumors, erythema papule scale, bullous, allergic and connective tissue problems that were brought to the attention of stakeholders by artificial intelligence [16]. Another similar multi-center study by Yong et al among populates of Singapore concluded that eczema was the most persistent skin disease (54.2\%) followed by tenia infection (20\%), cellulitis (11.4\%) and bullous pemphigoid (8.6\%) [17].

Identification of modifiable risk factors leading to rise in dermatological problems in our society are of paramount 
significance to our strategic planners in rational decision making for lessening the incidence of these cutaneous diseases. In our study, about 185 patients with diverse dermatological presentation got admitted in Dermatology ward during 2019 while admissions were diminished to total 138 during 2020. Males outnumbered females during 2019 while relatively less males were admitted during 2020 (Figure 2). Likewise, in a retrospective data analysis done by Sen a et al based on 2011 - 2014 record of admissions in Dermatology ward, males were comparatively more than females [18].

Many of the skin damaging and irritating effects nowadays are substantially attributed to ultraviolet radiations, oxides and particulate matter [19]. No doubt, exposure to environmental pollutants is also posing our population to the risk of developing skin diseases. Strategic planning to reduce Air Quality Index (AQI) by mitigating the proportion of pollutants is the need of hour in order to lower the occurrences of skin related issues globally. In present study, Average Length of Stay (ALOS) among our male and female patients during 2019 was $8.25 \pm 2.45$ days and $48.7 \pm 15.63$ respectively. Bed Occupancy rate during 2019 was $49 \%$. These Hospital statistics for 2020 were not computed due to closure of Dermatology department from April-July 2020. Bed Occupancy rate (BOR) of any healthcare facility is likely to exceed $100 \%$ in case of disaster or disease outbreak [20]. However, our study does not reflect any significant impact of COVID-19 on proportion of dermatological cases than those reported during 2019 (preCOVID era). Studies on dermatological statistics of other healthcare settings can facilitate us to great extent in situational analysis.

\section{Conclusion and Recommendations}

Erythroderma constituted the most prevalent skin disease of our community followed by pemphigus vulgaris and eczema; however, the frequency of these dermatological problems was not substantially influenced by COVID-19. The true picture in response to COVID pandemic can better be appraised by multi-center study.

\section{References}

1. Liu Y, Jain A, Eng C, Way DH, Lee K, et al. (2020) A deep learning system for differential diagnosis of skin diseases. Nat Med 26(6): 900-908.

2. Estébanez A, Pérez Santiago L, Silva E, Guillen Climent S, García Vázquez A, et al. (2020) Cutaneous manifestations in COVID-19: a new contribution. J Eur Acad Dermatol Venereol 34(6): e250-e251.
3. Almohideb M (2020) Epidemiological patterns of skin diseases in Saudi Arabia: A systematic review and meta-analysis. Dermatol Res Pract 10: $1-12$.

4. Rodriguez Hidalgo AJ, Pantaleon Y, Dios I, Falla D (2020) Fear of COVID-19, stress and anxiety in University undergraduate students: A predictive model for depression. Front Psychol 11: 591797.

5. Hay R, Flohr C (2021) Putting the burden of skin diseases on the global map. BJD 184(2): 189-190.

6. Zheng Y, Lai W (2020) Dermatology staff participate in fight against Covid-19 in China. J Eur Acad Dermatol Venereol 34(5): e210-E211.

7. Jamshidi P, Hajikhani B, Mirsaeidi M, Vahidnezhad H, Dadashi M, et al. (2021) Skin manifestations in COVID-19 patients: Are they indicators for disease severity? A systematic review. Front Med (Lausanne) 8: 634208.

8. Moan J, Grigalavicius M, Baturaite Z, Dahlback A, Juzeniene A (2015) The relationship between UV exposure and incidence of skin cancer. Photodermatol Photoimmunol Photomed 31(1): 26-35.

9. (2019) Science News. Skin diseases are more common than we think.

10. Seth D, Cheldize K, Brown D, Freeman F (2017) Global burden of skin disease: inequities and innovations. Curr Dermatol Rep 6(3): 204-210.

11. Centers for Disease Control and Prevention (CDC). Skin infections.

12. Jehangir F, Hasan A, Bashir F, Rahman N (2019) Lifestyle factors influencing the prevalence of skin diseases at a primary care center in Karachi. PJMD 8(1): 15-21.

13. Mponda K, Masenga J (2016) Skin diseases among elderly patients attending skin clinic at the regional Dermatology training centre, Northern Tanzania: A cross-sectional study. BMC ResNotes 9: 119.

14. Aman S, Nadeem M, Mahmood K, Ghafoor MB (2017) Pattern of skin diseases among patients attending a tertiary care hospital in Lahore, Pakistan. J Taibah Univ Med Sci 12(5): 392-396.

15. Boyers LN, Karimkhani C, Naghavi M, Sherwood D, Margolis DJ, et al. (2014) Global mortality from conditions with skin manifestations. J Am Acad Dermatol 71(6): 1137-1143.

16. Huang K, Jiang Z, Li Y, Wu Z, Wu X, et al. (2021) The classification of six common skin diseases based on Xiangya-Derm: Development of Chinese database for artificial intelligence. J Med Internet Res 23(9): e26025.

17. Yong AMY, Leung AJ, Chandran NS (2020) A Dermatology health services study of nursing homes in Singapore. Singapore Med J 61(12): 661-664.

18. Sen A, Chowdhury S, Poddar I, Bandyopadhyay D (2016) Inpatient Dermatology: Characteristics of patients and admissions in a tertiary level hospital in Eastern India. Indian J Dermatol 61(5): 561-564.

19. Drakaki E, Dessinioti C, Antoniou CV (2014) Air pollution and the skin. Front Environ Sci 2(11): 1-6.

20. Basic statistical data used in acute care facilities. Jones \& Bartlett Publishers. 
ISSN: 2574-1241

DOI: $10.26717 /$ BJSTR.2022.41.006530

Rizwana Shahid. Biomed J Sci \& Tech Res

(c) (P) This work is licensed under Creative BY Commons Attribution 4.0 License

Submission Link: https://biomedres.us/submit-manuscript.php

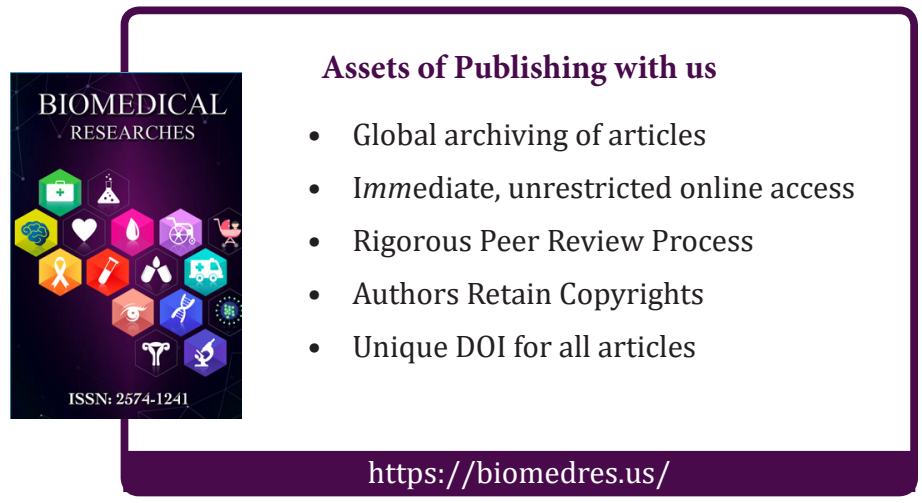

